\title{
Strongly modified plasmon-matter interaction with mesoscopic quantum emitters
}

\author{
Mads Lykke Andersen $^{1 \star}$, Søren Stobbe ${ }^{1}$, Anders Søndberg Sørensen ${ }^{2}$ and Peter Lodahl ${ }^{1 \star}$
}

Semiconductor quantum dots (QDs) provide useful means to couple light and matter in applications such as light-harvesting ${ }^{1,2}$ and all-solid-state quantum information processing ${ }^{3,4}$. This coupling can be increased by placing QDs in nanostructured optical environments such as photonic crystals or metallic nanostructures that enable strong confinement of light and thereby enhance the light-matter interaction. It has thus far been assumed that QDs can be described in the same way as atomic photon emitters-as point sources with wavefunctions whose spatial extent can be disregarded. Here we demonstrate that this description breaks down for QDs near plasmonic nanostructures. We observe an eightfold enhancement of the plasmon excitation rate, depending on QD orientation as a result of their mesoscopic character. Moreover, we show that the interaction can be enhanced or suppressed, determined by the geometry of the plasmonic nanostructure, consistent with a newly developed theory that takes mesoscopic effects into account. This behaviour has no equivalence in atomic systems and offers new opportunities to exploit the unique mesoscopic characteristics of QDs in the development of nanophotonic devices that use the increased light-matter interaction.

QDs are excellent single-photon sources ${ }^{5}$ and can store quantum bits for extended periods ${ }^{6}$, making them promising interconnects between light and matter in integrated quantum information networks ${ }^{7}$. An essential advantage of all-solid-state emitters compared with, for example, atomic emitters or molecules, is that they can be positioned deterministically and remain stationary ${ }^{8}$. This makes QD-based nanophotonic devices a promising technology for scalable many-qubit systems ${ }^{9}$. The term 'artificial atoms' has been coined for QDs owing to their discrete energy levels and their assumed atom-like interaction with light. It is now becoming clear that QDs in nanostructures lead to a number of surprises distinguishing them from atomic systems, including the recent observations of very broadband radiative coupling in cavity quantum electrodynamics ${ }^{10}$ and self-tuning of QD nanolasers ${ }^{11}$. Here, we present the first experimental observation and the theoretical explanation of a new mechanism to enhance the interaction between light and matter induced by the mesoscopic size of QDs. It gives rise to a strongly modified decay that is tailored by the size and shape of the QD electron-hole wavefunctions markedly enhancing the coupling of QDs to plasmonic nanostructures. The enhancement is mediated by strong electric field gradients and is not restricted to plasmonics but may generally appear in many nanophotonic structures. The efficient coupling of single emitters to plasmonic nanostructures is now being investigated intensively for various applications within nanophotonics and quantum optics ${ }^{12,13}$ enabling highly efficient single-photon sources ${ }^{14-17}$, single-photon
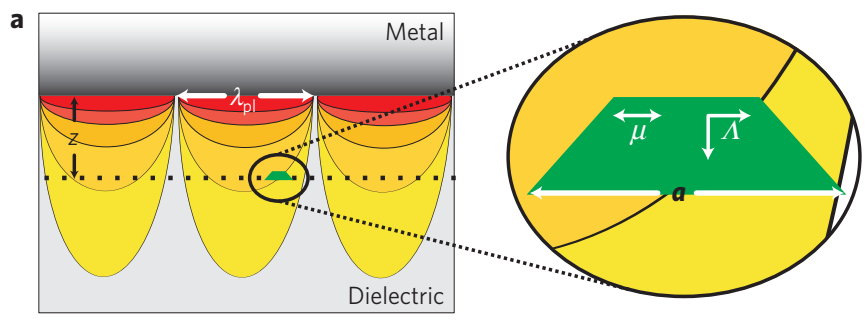

b

c
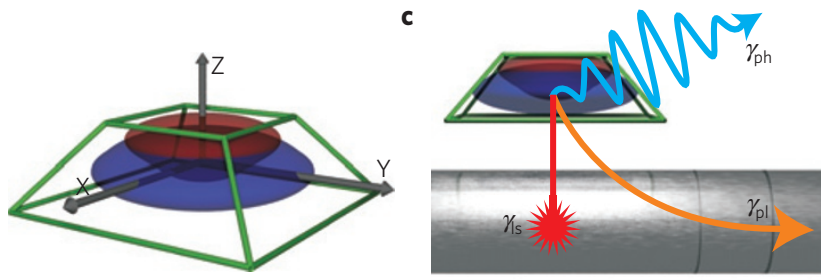

Figure 1 | Mesoscopic QDs in plasmonic nanostructures. a, Sketch of the studied system. A QD (green trapezoid) is placed a distance $z$ below a metal mirror. The lateral extension of a QD emitting at $1.2 \mathrm{eV}$ is typically $a=20 \mathrm{~nm}$. The plasmon wavelength is $\lambda_{\mathrm{pl}}=262 \mathrm{~nm}$ (figure is not to scale). The field amplitude of the plasmon decays exponentially away from the interface with a change of the electric field over the extension of the QD. The arrow over $\mu$ indicates the orientation of the point-dipole moment and the arrows over $\Lambda$ indicate the orientation of the first-order mesoscopic moment. $\mathbf{b}$, Boundaries of a QD (green frame) with the spatial extension of electron (blue) and hole (red) wavefunctions indicated inside. c, Sketch of a QD placed near a metallic structure. The QD can decay by emitting a photon $\left(\gamma_{\mathrm{ph}}\right)$, by exciting a propagating plasmon $\left(\gamma_{\mathrm{pl}}\right)$, by coupling to lossy modes in the metal $\left(\gamma_{\mathrm{s}}\right)$ or by intrinsic non-radiative recombination $\left(\gamma_{\mathrm{nr}}\right.$; not shown).

transistors $^{18}$ and subwavelength plasmon lasers ${ }^{19,20}$. In all of these applications, it is crucial to understand and enhance the interaction between light and matter, which is the essence of the work presented here.

Figure 1a illustrates the physical system under consideration: QDs are placed at a distance $z$ below a metallic mirror and the electromagnetic field associated with the surface-plasmon polariton resonance at the metal surface is varying over the extension of the QDs. The QDs are standard-sized $\left(\sim 20 \times 20 \times 6 \mathrm{~nm}^{3}\right)$ clusters of indium arsenide (InAs) embedded in gallium arsenide (GaAs) fabricated by molecular beam epitaxy. After excitation, the QDs trap single electrons and holes (Fig. 1b), which recombine through different channels with the following rates: excitation of plasmons $\gamma_{\mathrm{pl}}$, spontaneous emission of photons $\gamma_{\mathrm{ph}}$, non-radiative losses in the metal $\gamma_{\mathrm{ls}}$, or intrinsic non-radiative recombination in the

${ }^{1}$ DTU Fotonik, Department of Photonics Engineering, Technical University of Denmark, Ørsteds Plads bldg. 343, DK-2800 Kgs. Lyngby, Denmark, ${ }^{2}$ QUANTOP, Danish Quantum Optics Center and Niels Bohr Institute, DK-2100 Copenhagen Ø, Denmark. *e-mail: mlya@fotonik.dtu.dk; pelo@fotonik.dtu.dk. 


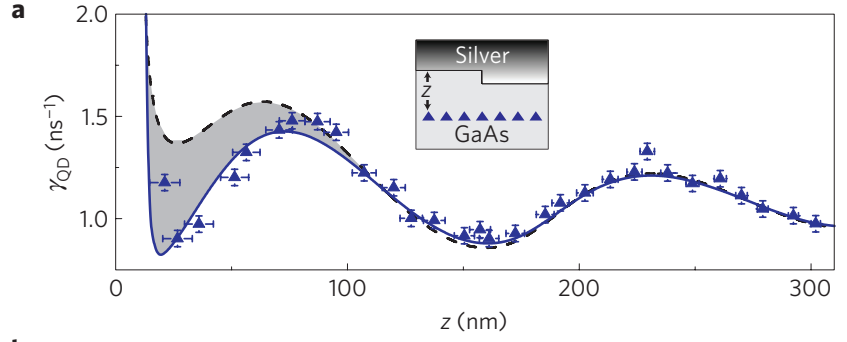

b
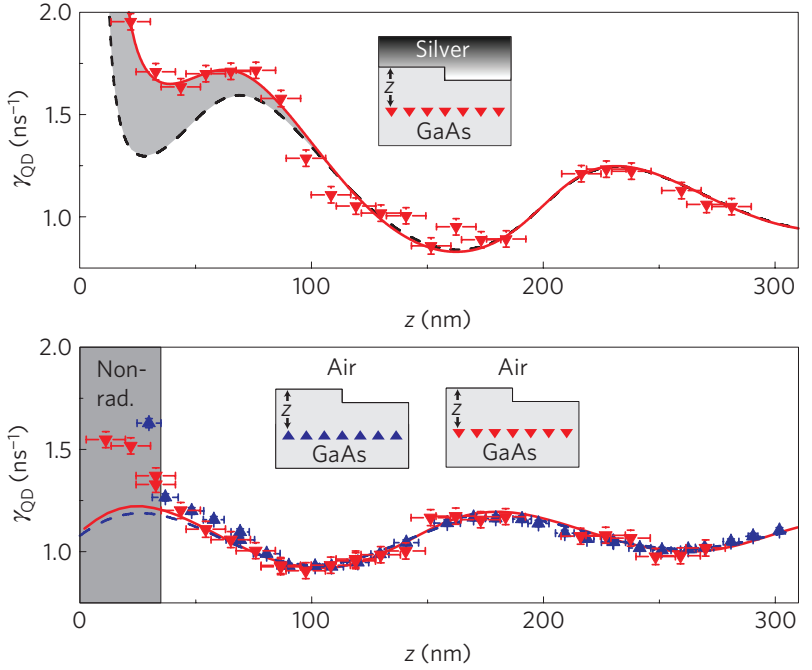

Figure 2 | Observation of the breakdown of the dipole approximation. $\mathbf{a}, \mathbf{b}$, Measured decay rates of QDs as a function of distance to the silver mirror for the direct (a) and inverted (b) structure at a wavelength of $\lambda=1,030 \mathrm{~nm}$. The dashed curves show the predicted variation for a point-dipole emitter. The solid curves show the theory for a mesoscopic emitter, and are found to match the experimental data very well. The insets show the orientation of the QDs relative to the silver mirrors for the two structures. c, Measured decay rates near a GaAs/air interface for $\lambda=1,030 \mathrm{~nm}$. Upwards (downwards)-pointing triangles are from the direct (inverted) structure. $\gamma_{\mathrm{rad}}$ and $\gamma_{\mathrm{nr}}$ are extracted by fitting the data from the inverted structure excluding the innermost points (indicated with dark shading). The error bars on all rates and positions represent one standard deviation and are deduced from repeated measurements.

QDs $\gamma_{\mathrm{nr}}$ (see Fig. 1c). The impact of the mesoscopic QD size on the radiative coupling to plasmonic nanostructures can be precisely assessed by employing a nanophotonic structure with wellunderstood optical properties. Here we employ a silver mirror with QDs positioned at precise distances from the surface, whereby the effects of the emitter and the environment can be unambiguously separated. This is not possible in complex structures such as photonic crystals ${ }^{21}$ or plasmonic nanowires ${ }^{15}$.

We have measured the decay rate of QDs $\left(\gamma_{\mathrm{QD}}\right)$ versus distance to the silver mirror ${ }^{22}$ (see Fig. 2a,b), allowing us to distinguish the various decay rates discussed above (see Supplementary Information for further details). Investigating two different orientations of the QDs relative to the silver mirror allows us to unambiguously prove the breakdown of the point-emitter description, that is, the so-called dipole approximation, which is found to be excellent for atoms, ions and molecules. In the first sample (the direct structure), the apex of the QDs points towards the silver mirror, whereas in the second sample (the inverted structure) it points away (see insets of Fig. 2). A point-dipole source would radiate identically in the direct and inverted structures, and the expected decay rate for such an emitter is shown in Fig. 2. We observe that for short distances to the silver mirror, the measured decay rates deviate significantly from the point-dipole theory; that is, significantly slower (faster) QD

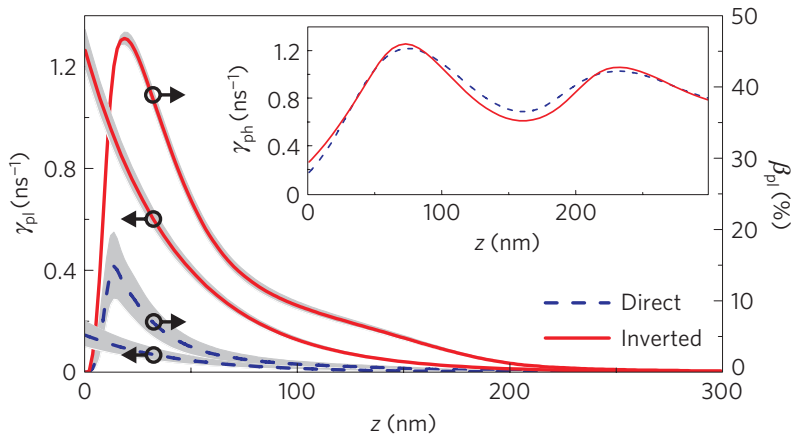

Figure 3 | Influence of mesoscopic effects on decay rates. Rate of decay to plasmons ( $\gamma_{\mathrm{pl}}$, left axis) and associated efficiency $\left(\beta_{\mathrm{pl}}\right.$, right axis) as a function of distance to the silver mirror, $z$. Solid (dashed) curves are for the inverted (direct) structure. The grey shading indicates the uncertainties deduced from the standard deviations of the extracted mesoscopic moments $(\Lambda)$. The inset shows the coupling to photons $\left(\gamma_{\mathrm{ph}}\right)$ for both structures; their difference is due to different distances to the backside of the wafer (see Supplementary Information).

decay dynamics is observed for the direct (inverted) structure compared with the expectations for a dipole emitter. These deviations originate from the mesoscopic nature of QDs, implying that the electron and hole wavefunctions are spatially extended, as pictured in Fig. 1b. The experimental data are well explained by our theory, which accounts for the mesoscopic size of the QDs, and we can independently rule out alternative mechanisms, for example, carrier tunnelling, non-radiative processes, light-hole contribution to the dipole moment, or quantum-confined Stark shifts ${ }^{23}$ of the QD energy levels induced by the metallic surface (see Supplementary Information). The deviations from dipole theory are observed only for QDs positioned closer than $\sim 100 \mathrm{~nm}$ from the mirror, which is equal to the length scale of the plasmonic penetration depth into the GaAs substrate. The observed variations in decay rate directly illustrate that the mesoscopic character of the QDs strongly influences the coupling to plasmons and can be employed as a resource to either suppress (direct structure) or promote (inverted structure) the excitation of plasmons.

The modified excitation of plasmons stems from the mesoscopic dimensions of the QD (ref. 24). Here we develop a new model for spontaneous emission from mesoscopic QDs that includes the spatial extent and asymmetry of the QD wavefunctions. By expanding the interaction to first order around the centre of the QD, we obtain the total decay rate

$$
\gamma_{\mathrm{QD}}(z)=\gamma_{\mathrm{pd}}(z)+\xi_{\mathrm{me}}(z)
$$

where $\gamma_{\mathrm{pd}}(z)$ is the familiar point-dipole contribution, which depends on the transition dipole moment proportional to $\mu_{x}=\left\langle\Psi_{\mathrm{h}}\left|\hat{p}_{x}\right| \Psi_{\mathrm{e}}\right\rangle$, and $\xi_{\mathrm{me}}(z)$ is the first-order mesoscopic contribution, which is characterized by the moment $\Lambda_{z, x}=\left\langle\Psi_{\mathrm{h}}\left|\hat{p}_{z} \hat{x}\right| \Psi_{\mathrm{e}}\right\rangle$, in the following denoted $\mu$ and $\Lambda$. Here $\Psi_{\mathrm{e}}\left(\Psi_{\mathrm{h}}\right)$ is the wavefunction of an electron (hole) trapped in the QD, and $\hat{x}$ and $\hat{p}_{z}$ are position and momentum operators for the $x$ and $z$ directions, respectively. $\Lambda$ is an intrinsic property of the QD and is determined by the size and geometry of the electron and hole wavefunctions.

Owing to their mesoscopic dimensions and asymmetric confinement potential QD wavefunctions are extended and asymmetric ${ }^{23}$, as sketched in Fig. 1b. The mesoscopic contribution to the decay rate $\xi_{\mathrm{me}}(z)$ depends not only on $\Lambda$ but also on the optical field gradient, which is large for plasmonic modes (see Fig. 1a). The two contributions to the decay rate in equation (1) combine coherently and therefore can either add or subtract depending on the specific 


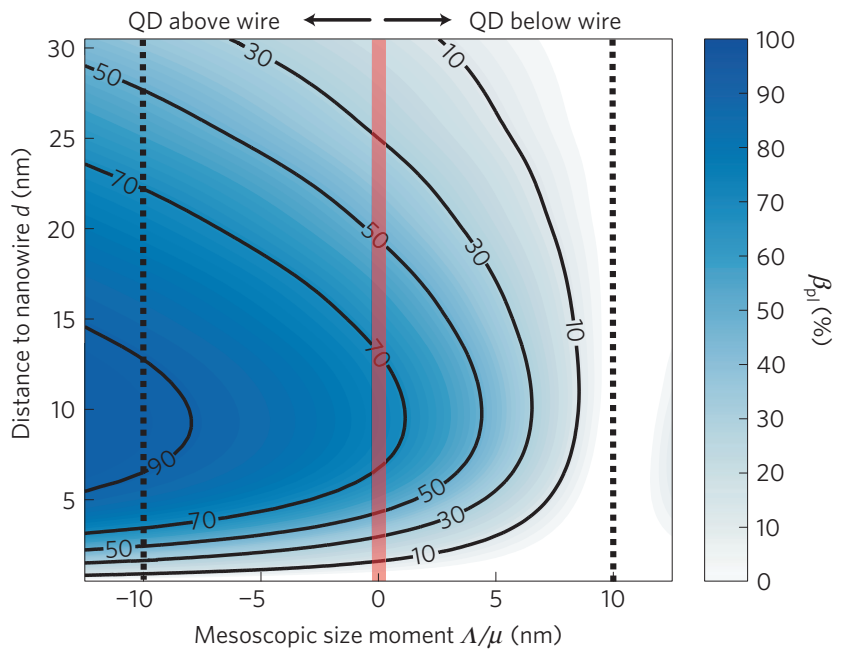

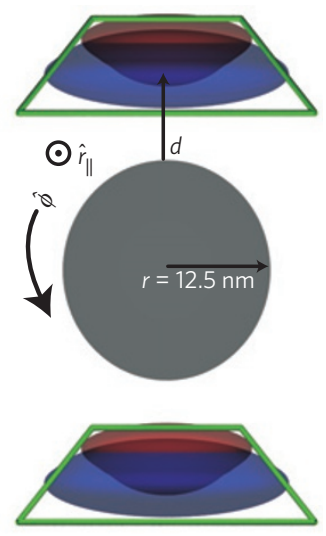

Figure 4 | Efficiency of a nanoplasmonic single-photon source with a mesoscopic emitter. a, Plasmon generation efficiency $\beta_{\mathrm{pl}}$ for a QD in GaAs near a

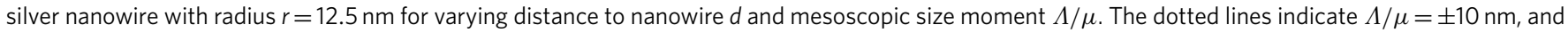
are representative for the experiment. $\mathbf{b}$, Sketch of a nanowire with $r=12.5 \mathrm{~nm}$ and mesoscopic QDs positioned a distance $d$ from the surface. The dipole moment of the modelled QD is oriented at $45^{\circ}$ to both the parallel $\left(\hat{r}_{\|}\right)$and azimuthal $(\hat{\phi})$ directions. Negative (positive) $\Lambda / \mu$ corresponds to a QD positioned above (below) the nanowire.

nanophotonic structure surrounding the QD, as was observed for the direct and inverted mirror structures in the data of Fig. 2a,b. This previously unknown effect has no counterpart in atomic systems where the higher-order interactions between light and atoms are restricted by selection rules, that is, coexistence of the first higher-order and dipole transitions is prohibited by the symmetry of the atomic potential. The moment $\Lambda$ contains both electric quadrupolar and magnetic dipolar terms and would in the case of atomic-like emitters vanish on electric-dipole transitions ${ }^{25,26}$. We observe that for QD emitters the higher-order processes can strongly modify the dipole transition owing to the mesoscopic and asymmetric nature of the wavefunctions, thereby enhancing the light-matter interaction strength significantly.

To enable a direct comparison to dipole theory, the intrinsic radiative decay rate $\gamma_{\mathrm{rad}}$ and non-radiative decay rate $\gamma_{\mathrm{nr}}$ of the QDs are extracted from measurements of the total decay rate as a function of distance to a GaAs/air interface for the direct and inverted samples before evaporating silver (see Fig. 2c). From our theory we predict a negligible effect of the mesoscopic moments because field gradients are small near the GaAs/air interface and indeed we find that these measurements are well described by the point-dipole term alone. We extract $\gamma_{\mathrm{rad}}=0.88 \mathrm{~ns}^{-1}$ and $\gamma_{\mathrm{nr}}=0.19 \mathrm{~ns}^{-1}$. We observe enhanced decay rates very near the GaAs/air interfaces $(z<35 \mathrm{~nm})$, that notably, are independent of QD orientation. They are attributed to tunnelling to the surface or coupling to a lossy surface layer ${ }^{27}$, caused by, for example, dangling bonds, and these data points are disregarded in the analysis. For the mirror samples the surface is terminated with silver, whereby the interface becomes well controlled and such effects are not observed. Consequently, the new theory models the experimental data of Fig. 2a,b very well for both the direct and inverted structure for all distances. From the comparison we extract the experimental values $\Lambda=(9.8 \pm 1.4) \times 10^{-33} \mathrm{~kg} \mathrm{~m}^{2} \mathrm{~s}^{-1}\left(\Lambda=(-6.5 \pm 0.8) \times 10^{-33} \mathrm{~kg} \mathrm{~m}^{2} \mathrm{~s}^{-1}\right)$ for the direct (inverted) structure from the experimental value of $\mu$ obtained from the data of Fig. 2c. The observed change of the sign of $\Lambda$ stems from the opposite orientation of the QDs relative to the plasmonic field and constitutes the telltale of the mesoscopic effects.

From our comparison with theory we can extract the rate of excitation of plasmons $\gamma_{\mathrm{pl}}(z)$, which should be maximized in fast quantum plasmonic devices (see Fig. 3). A pronounced difference of the plasmon excitation rate by a factor of eight is observed between the two structures. In contrast, the spontaneous-emission rate of photons $\gamma_{\mathrm{ph}}(z)$ (inset of Fig. 3) is similar for the two different structures. These observations can be explained from the fact that plasmon modes have strong electric-field gradients near the metal mirror, thereby enhancing the influence of the mesoscopic QD effects. We note that the extracted mesoscopic and point-dipole contributions to the plasmon excitation rate are of equal magnitude. Therefore, the mesoscopic contribution is so pronounced that the approximation of keeping only first-order mesoscopic moments is pushed to the limit of validity, which could account for the slight difference in the magnitudes of $\Lambda$ between the two data sets. The figure-of-merit of a quantum plasmonic device is the $\beta$-factor expressing the probability that a QD excites a single plasmon: $\beta_{\mathrm{pl}}(z)=\gamma_{\mathrm{pl}}(z) / \gamma_{\mathrm{QD}}(z)$, which is plotted in Fig. 3. The $\beta$-factor is enhanced as a result of the mesoscopic effects reaching $46 \%$ for the inverted sample as opposed to $15 \%$ for the direct sample where it is suppressed. These observations illustrate the potential of using the intrinsic mesoscopic properties of QDs in combination with careful engineering of the electromagnetic environment to strongly enhance the coupling to plasmons.

As a potential application of the observations, we further investigate the use of mesoscopic QDs for improving plasmonnanowire single-photon sources ${ }^{14}$ (see Fig. $4 \mathrm{~b}$ ). For a small wire radius $(r=12.5 \mathrm{~nm})$, only a single strongly confined plasmon exists inducing very strong field gradients; that is, mesoscopic QD effects are expected to be very pronounced. We note that structures of this size can readily be fabricated by electron beam lithography or chemical synthesis. We calculate $\gamma_{\mathrm{pl}}(z)$ versus distance to the nanowire for varying ratios of $\Lambda / \mu$, corresponding to QDs with various amounts of mesoscopic character, and for two different orientations of the QD relative to the nanowire. The coupling to photons is assumed constant and the coupling to lossy modes is treated in the dipole approximation (see Supplementary Information). The resulting plasmonic coupling efficiency $\beta_{\mathrm{pl}}$ in this simplified model is shown in Fig. 4a. Very strong dependencies on both distance and $\Lambda / \mu$ are observed. For a fixed distance of $d=10 \mathrm{~nm}$, we find that the efficiency for a point-dipole source (that is, $\Lambda=0)$ is $\beta_{\mathrm{pl}}=75 \%$. This number can be enhanced substantially to $\beta_{\mathrm{pl}}=92 \%$ assuming the experimental value of $\Lambda / \mu \approx-10 \mathrm{~nm}$ for a QD placed near the nanowire (see Fig. $4 \mathrm{~b}$ ). On the other 
hand, the same QD oriented upside-down relative to the nanowire $(\Lambda / \mu \approx 10 \mathrm{~nm})$ would couple only weakly to the nanowire with $\beta_{\mathrm{pl}}<1 \%$. These results demonstrate the very pronounced effects of including the naturally occurring mesoscopic contribution to the QD decay and that it can be employed for improving the efficiency of plasmonic nanophotonic devices.

We have demonstrated that the interaction between QDs and plasmonic nanostructures can be understood only by taking the mesoscopic size of the QDs into account. Our findings are not limited to plasmonic structures and are expected to be of relevance for a range of nanophotonic structures where strong field gradients are abundant. Thus, mesoscopic QD effects are anticipated to be of importance for spontaneous-emission control in photonic crystals ${ }^{21}$, dielectric-waveguide single-photon sources $^{28}$, and in cavity quantum electrodynamics ${ }^{10}$ in particular when employing large QD emitters that are now being intensively investigated for their prospective large oscillator strength ${ }^{29}$. Our conclusions are surprising because the point-dipole approximation has been uncritically adopted in the literature to describe lightmatter interaction between QDs and nanophotonic structures. Importantly, the mesoscopic effects are very pronounced and may be employed as a resource to enhance light-matter interaction, which is required in a diverse range of scientific fields ranging from quantum information science and quantum computing to energy-harvesting devices.

\section{Received 22 July 2010; accepted 1 November 2010; published online 19 December 2010}

\section{References}

1. Atwater, H. A. \& Polman, A. Plasmonics for improved photovoltaic devices. Nature Mater. 9, 205-213 (2010).

2. Nozik, A. J. Quantum dot solar cells. Physica E 14, 115-120 (2002).

3. Fattal, D., Diamanti, E., Inoue, K. \& Yamamoto, Y. Quantum teleportation with a quantum dot single photon source. Phys. Rev. Lett. 92, 037904 (2004).

4. Ladd, T. D. et al. Quantum computers. Nature 464, 45-53 (2010).

5. Michler, P. et al. A quantum dot single-photon turnstile device. Science 290, 2282-2285 (2000).

6. Taylor, J. M., Marcus, C. M. \& Lukin, M. D. Long-lived memory for mesoscopic quantum bits. Phys. Rev. Lett. 90, 206803 (2003).

7. Loss, D. \& DiVincenzo, D. P. Quantum computation with quantum dots. Phys. Rev. A 57, 120-126 (1998).

8. Schmidt, O. G. (ed.) Lateral Alignment of Epitaxial Quantum Dots (Springer, 2007)

9. O'Brien, J. L., Furusawa, A. \& Vuckovic, J. Photonic quantum technologies. Nature Photon. 3, 687-695 (2009).

10. Hennessy, K. et al. Quantum nature of a strongly coupled single quantum dot-cavity system. Nature 445, 896-899 (2007).

11. Strauf, S. et al. Self-tuned quantum dot gain in photonic crystal lasers. Phys. Rev. Lett. 96, 127404 (2006).

12. Gramotnev, D. K. \& Bozhevolnyi, S. I. Plasmonics beyond the diffraction limit. Nature Photon. 4, 83-91 (2010).

13. Schuller, J. A. et al. Plasmonics for extreme light concentration and manipulation. Nature Mater. 9, 193-204 (2010).
14. Chang, D. E., Sørensen, A. S., Hemmer, P. R. \& Lukin, M. D. Quantum optics with surface plasmons. Phys. Rev. Lett. 97, 053002 (2006).

15. Akimov, A. V. et al. Generation of single optical plasmons in metallic nanowires coupled to quantum dots. Nature 450, 402-406 (2007).

16. Jun, Y. C., Kekatpure, R. D., White, J. S. \& Brongersma, M. L. Nonresonant enhancement of spontaneous emission in metal-dielectric-metal plasmon waveguide structures. Phys. Rev. B 78, 153111 (2008).

17. Kolesov, R. et al. Wave particle duality of single surface plasmon polaritons. Nature Phys. 5, 470-474 (2009).

18. Chang, D. E., Sørensen, A. S., Demler, E. A. \& Lukin, M. D. A single-photon transistor using nanoscale surface plasmons. Nature Phys. 3, 807-812 (2007).

19. Bergman, D. J. \& Stockman, M. I. Surface plasmon amplification by stimulated emission of radiation: Quantum generation of coherent surface plasmons in nanosystems. Phys. Rev. Lett. 90, 027402 (2003).

20. Noginov, M. A. et al. Demonstration of a spaser-based nanolaser. Nature 460, 1110-1112 (2009)

21. Lodahl, P. et al. Controlling the dynamics of spontaneous emission from quantum dots by photonic crystals. Nature 430, 654-657 (2004).

22. Drexhage, K. H. Influence of a dielectric interface on fluorescence decay time. J. Lumin. 1-2, 693-701 (1970).

23. Finley, J. J. et al. Quantum-confined Stark shifts of charged exciton complexes in quantum dots. Phys. Rev. B 70, 201308 (2004).

24. Jun Ahn, K. \& Knorr, A. Radiative lifetime of quantum confined excitons near interfaces. Phys. Rev. B 68, 161307 (2003).

25. Zurita-Sánchez, J. R. \& Novotny, L. Multipolar interband absorption in a semiconductor quantum dot. I. Electric quadrupole enhancement. J. Opt. Soc. Am. B 19, 1355-1362 (2002).

26. Rukhlenko, I. D. et al. Spontaneous emission of guided polaritons by quantum dot coupled to metallic nanowire: Beyond the dipole approximation. Opt. Express 17, 17570-17581 (2009).

27. Johansen, J. et al. Size dependence of the wavefunction of self-assembled InAs quantum dots from time-resolved optical measurements. Phys. Rev. B. 77, 073303 (2008).

28. Lund-Hansen, T. et al. Experimental realization of highly efficient broadband coupling of single quantum dots to a photonic crystal waveguide. Phys. Rev. Lett. 101, 113903 (2008).

29. Andreani, L. C., Panzarini, G. \& Gérard, J-M. Strong-coupling regime for quantum boxes in pillar microcavities: Theory. Phys. Rev. B 60, 13276-13279 (1999).

\section{Acknowledgements}

We would like to thank Y. Chen for supplying numerical code for the finite-element calculations and M. Wubs and J. M. Hvam for careful comments on the manuscript. We gratefully acknowledge financial support from the Villum Kann Rasmussen Foundation, The Danish Council for Independent Research (Natural Sciences and Technology and Production Sciences) and the Danish National Research Foundation.

\section{Author contributions}

M.L.A. and S.S. fabricated the samples and developed the concepts. M.L.A. carried out the experiment, analysed the data and implemented the theoretical model. A.S.S suggested the use of the inverted structure and supported the theoretical work. M.L.A. and P.L. wrote the Letter. P.L. initiated and supervised the project. All authors provided detailed comments on the manuscript.

\section{Additional information}

The authors declare no competing financial interests. Supplementary information accompanies this paper on www.nature.com/naturephysics. Reprints and permissions information is available online at http://npg.nature.com/reprintsandpermissions. Correspondence and requests for materials should be addressed to M.L.A. or P.L. 